\title{
Possible Quasi-One-Dimensional Fermi Surface in $\mathrm{La}_{2-x} \mathrm{Sr}_{x} \mathrm{CuO}_{4}$
}

\author{
Hiroyuki Yamase $^{1}$ and Hiroshi Kohno ${ }^{2}$ \\ ${ }^{1}$ Institute for Solid State Physics, University of Tokyo, 7-22-1 Roppongi, Minato-ku, Tokyo 106-8666 \\ ${ }^{2}$ Graduate School of Engineering Science, Osaka University, Toyonaka, Osaka 560-8531
}

(Received September 14, 1999)

\begin{abstract}
To reconcile the two experimental findings on $\mathrm{La}_{2}{ }_{-} \mathrm{Sr}_{x} \mathrm{CuO}_{4}$, namely, Fermi surface (FS) observed by angle-resolved photoemission spectroscopy and sharp incommensurate magnetic peaks by neutron scattering, we propose a picture that a quasi-one-dimensional FS (q-1dFS) is realized in each $\mathrm{CuO}_{2}$ plane whose q-1d direction alternates along the $c$-axis.
\end{abstract}

KEYWORDS: LSCO, $t-J$ model, incommensurate antiferromagnetism, Fermi surface, ARPES, neutron scattering

Early studies by neutron scattering revealed the material dependence of low-energy spin excitations in high- $T_{\mathrm{c}}$ cuprates: $\mathrm{La}_{2-x} \mathrm{Sr}_{x} \mathrm{CuO}_{4}(\mathrm{LSCO})^{1)}$ in the metallic region shows sharp incommensurate antiferromagnetic (IC-AF) peaks at wavevectors $(\pi \pm 2 \pi \eta, \pi)$ and $(\pi, \pi \pm 2 \pi \eta)$, where $\eta>0$ is called incommensurability, while in $\mathrm{YBa}_{2} \mathrm{Cu}_{3} \mathrm{O}_{6+y}(\mathrm{YBCO})^{2,3)}$ there is a single broad commensurate structure at wavevector $(\pi, \pi)$. It was pointed out theoretically ${ }^{4}$ that such material dependence could be understood in terms of the shape of the Fermi surface (FS): in the calculation based on the $t-J$ model, ${ }^{5)}$ a diamond-shaped 'electron-like' FS centered at $(0,0)$ suggested an IC-AF fluctuation, while an almost circular 'hole-like' FS centered at $(\pi, \pi)$ suggested a commensurate one. The former has been presumed to be applicable to LSCO, and the latter to YBCO.

However, angle-resolved photoemission spectroscopy (ARPES $)^{6)}$ performed recently on LSCO has revealed some segments of the FS, which looks more consistent with the FS of YBCO-type rather than LSCO-type, at low doping rates, $\delta .{ }^{7)}$ This experimental finding has raised a serious question as to whether low-energy spin excitation in LSCO can be understood in terms of fermiology.

There is an interesting indication, ${ }^{8)}$ based on the fluctuation exchange (FLEX) approximation on the Hubbard model, that even with YBCO-type FS, the IC peak can be reproduced, suggesting that fermiology is sufficient for understanding the IC-AF fluctuation. However, such IC peaks are not isolated but are connected with each other with substantial spectral weight at wavevector $(\pi, \pi)$, resulting in an essentially commensurate peak with an IC sub- 
structure. Thus, YBCO-type FS consistent with ARPES data seems incompatible with the sharp IC-AF peaks observed by inelastic neutron scattering.

How can we reconcile these two experimental findings, namely those by ARPES and neutron scattering? One solution may be to abandon the connection of the IC-AF fluctuation to fermiology. Actually, the currently growing picture ${ }^{9-11)}$ of 'charge stripe' may offer another route to the IC peaks without recourse to fermiology.

In this paper, however, we point out that we can construct a consistent picture between fermiology and sharp IC-AF peaks in LSCO if we assume a quasi-one-dimensional FS (q1dFS), not a two-dimensional FS (2dFS), in each $\mathrm{CuO}_{2}$ plane. This assumption is based on our recent theoretical result ${ }^{12)}$ that the $2 \mathrm{~d} t-J$ model treated in the mean-field approximation has an instability towards forming a q-1d band. (Remarkably, this instability occurs even in homogeneous charge distribution, and we assume this charge homogeneity in this paper.) Deferring the details of the microscopic origin of the q-1d band elsewhere, ${ }^{13)}$ we here show that the FS observed by $\mathrm{ARPES}^{6)}$ can be regarded as consisting of two orthogonal q-1dFSs; we can fit the former by the latter at each doping rate. We then calculate the doping dependence of $\eta$, which is found to improve quantitatively the result calculated in the previous scheme.5) We also explore the effects of orthorhombicity of the crystal structure on the wavevector of IC-AF, motivated by recent elastic neutron scattering studies. ${ }^{14,15)}$

Let us first assume that either of two kinds of q-1dFSs, q-1dFS(x) or q-1dFS(y), is realized in each $\mathrm{CuO}_{2}$ plane and is stacked alternately along the $c$-axis as shown in Fig. 1. This assumption of alternate stacking may be justified by the fact that the $\mathrm{q}-1 \mathrm{~d}$ band couples to the soft phonon mode ${ }^{16)}$ related to the instability toward the low-temperature tetragonal (LTT) structure.

We first show that our q-1dFS picture does not conflict with ARPES data ${ }^{6)}$ that imply a 2dFS. Since there is a small, but not negligible, interlayer hopping integral, $\tilde{t}_{z},{ }^{17)}$ two dispersions, $\xi_{\boldsymbol{k}}^{\mathrm{A}}$ and $\xi_{\boldsymbol{k}}^{\mathrm{B}}$, each forming $\mathrm{q}-1 \mathrm{dFS}(\mathrm{x})$ and $\mathrm{q}-1 \mathrm{dFS}(\mathrm{y})$, respectively, hybridize to become

$$
\xi_{\boldsymbol{k}}^{ \pm}=\frac{\xi_{\boldsymbol{k}}^{\mathrm{A}}+\xi_{\boldsymbol{k}}^{\mathrm{B}} \pm \sqrt{\left(\xi_{\boldsymbol{k}}^{\mathrm{A}}-\xi_{\boldsymbol{k}}^{\mathrm{B}}\right)^{2}+4 \epsilon_{\boldsymbol{k}}^{2}}}{2}
$$

where

$$
\epsilon_{\boldsymbol{k}}=\tilde{t}_{z} \cos \frac{k_{x}}{2} \cos \frac{k_{y}}{2} \cos \frac{k_{z}}{2} .
$$

The form factor, $\cos \frac{k_{x}}{2} \cos \frac{k_{y}}{2} \cos \frac{k_{z}}{2}$, comes from the fact that $\mathrm{Cu}$ sites on adjacent $\mathrm{CuO}_{2}$ planes are relatively displaced by $[1 / 2,1 / 2,1 / 2]$. The resulting FS consists of the 'outer FS' 
formed by $\xi_{\boldsymbol{k}}^{-}$and the 'inner FS' by $\xi_{\boldsymbol{k}}^{+}$as shown in Fig. 2, where the band width of $\epsilon_{\boldsymbol{k}}$ is taken to be about 0.1 times that of the $\xi_{\boldsymbol{k}}^{\mathrm{A}}\left(\right.$ or $\xi_{\boldsymbol{k}}^{\mathrm{B}}$ ). Note that $\epsilon_{\boldsymbol{k}}=0$ at $(\pi, 0)$ and $(0, \pi)$, where no dispersion appears along $k_{z}$. Since the ARPES spectrum has a very broad peak even near $(\pi, 0)$ and $(0, \pi)$, the effect of the dispersion along $k_{z}$ will be sufficient to blur the 'inner FS' and the 'outer FS' except near $(\pi, 0)$ and $(0, \pi)$, if the ARPES spectrum is somewhat integrated along the $k_{z}$-direction. This interpretation is consistent with the ARPES data, ${ }^{6)}$ in which the FS is always detected near $(\pi, 0)$ and $(0, \pi)$ for $0.05 \leq \delta \leq 0.30$, but not near $(\pi / 2, \pi / 2)$ especially below $\delta \sim 0.15$. Therefore, we propose that the FS observed by ARPES comes from the $\mathrm{q}-1 \mathrm{dFS}(\mathrm{x})$ and the $\mathrm{q}-1 \mathrm{dFS}(\mathrm{y})$. We note here a recent finding in $\operatorname{ARPES}{ }^{18)}$ that momentum distribution, $n(\boldsymbol{k} ; \Lambda)$, depends on the range, $\Lambda$, of energy integration near the Fermi energy. This dependence will also be understood as coming from effects of $\tilde{t}_{z}$ : for $\Lambda<\left|\tilde{t}_{z}\right|, n(\boldsymbol{k} ; \Lambda)$ reflects effects of the dispersion along $k_{z}$, while it does not for $\Lambda \gg\left|\tilde{t}_{z}\right|$. (These discussions hold even if we consider $d$-wave singlet pairing in eq. (1).)

In order to determine the $\mathrm{q}-1 \mathrm{~d}$ dispersions, $\xi_{\boldsymbol{k}}^{\mathrm{A}}$ and $\xi_{\boldsymbol{k}}^{\mathrm{B}}$, we use the previous mean field theory $^{5)}(\mathrm{MFT})$ of the $2 \mathrm{~d} t-J$ model on a square lattice. We set temperature as $T=0.01 J$ where the thermal smearing of the FS can be neglected. We take the parameters as $t / J=4$ and $t^{\prime} / t=-1 / 6$, where $J>0$ is the nearest neighbor (n.n.) superexchange coupling, $t$ is the first n.n. hopping integral and $t^{\prime}$ is the second one. These parameters are chosen so as to reproduce the observed FS at $\delta=0.30$ where the whole shape of the FS has been clarified. ${ }^{6)}$ Previously, ${ }^{5)}$ the dispersion for (spin) fermions was given by $E_{\boldsymbol{k}}=\sqrt{\xi_{\boldsymbol{k}}^{2}+\left|\Delta_{\boldsymbol{k}}\right|^{2}}$, where $\Delta_{\boldsymbol{k}}=-\frac{3}{4} \Delta_{0}\left(\cos k_{x}-\cos k_{y}\right)\left(\Delta_{0}\right.$ is the singlet order parameter $)$ and

$$
\xi_{\boldsymbol{k}}=-2\left(F_{x} \cos k_{x}+F_{y} \cos k_{y}+F_{\|}^{\prime} \cos \left(k_{x}+k_{y}\right)+F_{\perp}^{\prime} \cos \left(k_{x}-k_{y}\right)\right)-\mu,
$$

with $F_{x}=F_{y}, F_{\|}^{\prime}=F_{\perp}^{\prime}$ and $\mu$ being the chemical potential. The values of $F_{x}, F_{\|}^{\prime}, \mu$ and $\Delta_{0}$ were determined numerically by minimizing the free energy. The resulting FS was used for discussing LSCO.5) We call this FS 'previous FS', because recent ARPES'6) has revealed that the 'previous FS' is incompatible with the observed FS especially for low $\delta$. Thus, in this paper, we reduce the value of $F_{y}$ as

$$
F_{y}=\alpha F_{x}
$$

for the q-1dFS(x), retaining for the other parameters, $F_{x}, F_{\|}^{\prime}, F_{\perp}^{\prime}, \mu$ and $\Delta_{0}$, the values of the previous MFT. ${ }^{5)}$ (For the $\mathrm{q}-1 \mathrm{dFS}(\mathrm{y})$ we take $F_{x}=\alpha F_{y}$.) We choose the value of $\alpha$ to adjust the $\mathrm{q}-1 \mathrm{dFS}(\mathrm{x})$ near $(0, \pi)$ to the observed FS segments. The obtained values are $\alpha=0.75,0.78,0.85,0.92$ and 1.0 for $\delta=0.05,0.10,0.15,0.22$ and 0.30 , respectively: the 
band anisotropy decreases with doping and the 2dFS is realized at $\delta=0.30$.

Next, we study the wavevector of IC-AF fluctuation in the state with the q-1dFS. For simplicity, we neglect the interlayer coupling, $\tilde{t}_{z}$, and treat the $\mathrm{q}-1 \mathrm{dFS}(\mathrm{x})$ and the $\mathrm{q}-1 \mathrm{dFS}(\mathrm{y})$ independently. Since the energy scale of the IC-AF fluctuation is about 0.02J $(2 \sim 3$ $\mathrm{meV}$ ) experimentally, ${ }^{1)}$ which is much smaller than $J$, it will be reasonable to estimate the wavevector of IC-AF fluctuation from the peak position of the static magnetic susceptibility, $\chi_{0}(\boldsymbol{q})$, for non-interacting fermions. In Fig. 3, we plot $\chi_{0}(\boldsymbol{q})$ for the $\mathrm{q}-1 \mathrm{dFS}(\mathrm{x})$ along $(\pi, \pi / 2) \rightarrow(\pi, \pi) \rightarrow(\pi / 2, \pi)$ at $\delta=0.15$ as a typical example. We see two IC peaks whose heights are comparable: the IC peak along $(\pi, \pi / 2) \rightarrow(\pi, \pi)$ is located at $\left(\pi, \pi-2 \pi \eta^{y}\right)$ and further deviates from $(\pi, \pi)$ relative to that for the 'previous FS', whereas the other peak at $\left(\pi-2 \pi \eta^{x}, \pi\right)$ remains at almost the same position as that for the 'previous FS'. (Note that IC peaks are $2 \mathrm{~d}$-like rather than $1 \mathrm{~d}-$-like even in the state of the q-1dFS.) For the $\mathrm{q}-1 \mathrm{dFS}(\mathrm{y})$, $\chi_{0}(\boldsymbol{q})$ is obtained by the $90^{\circ}$ rotation from that for the q-1dFS(x). Figure 4(a) schematically shows the wavevector at which $\chi_{0}(\boldsymbol{q})$ takes a peak for each q-1dFS. In Fig. 4(b), $\eta^{x}$ and $\eta^{y}$ are shown as a function of $\delta$ : compared with the results for the 'previous FS' (open triangles), the values of $\eta^{y}$ (filled circles) become much larger especially in $0.10 \lesssim \delta \lesssim 0.15$, while the values of $\eta^{x}$ (filled squares) are almost unchanged.

Since inelastic neutron scattering data have not been analyzed in terms of two incommensurabilities, $\eta^{x}$ and $\eta^{y}$, comparison of the present results directly with the experimental $\eta-\delta$ curve $^{1)}$ (indicated by crosses in Fig. 4(b)) cannot be done in a straightforward manner. (We leave as a future problem the recently observed 'diagonal IC peak' (located at $(\pi \pm 2 \pi \eta, \pi \pm 2 \pi \eta))$ at $\left.\delta=0.05 .^{19)}\right)$ Nonetheless, the results for the q-1dFS seem valid in the following aspects. First, through the calculation of the $\operatorname{Im}_{\chi_{0}}(\boldsymbol{q}, \omega)$ at $\omega=0.01 \mathrm{~J}$ and $T=0.01 \mathrm{~J}$, we find that in contrast to the $2 \mathrm{dFS},{ }^{8)}$ the $\mathrm{q}-1 \mathrm{dFS}$ yields sharp IC peaks even at low $\delta$, which is consistent with experiments. ${ }^{1)}$ A preliminary result for $\delta=0.10$ is shown in Fig. 5. Second, the values of the $\eta^{y}$ for the $\mathrm{q}-1 \mathrm{dFS}(\mathrm{y})$ are closer to the experimental values than those for the 'previous FS'.

There is another interesting phenomenon, which we call the 'shift', that the IC peaks do not lie exactly on the symmetry axes $\left(k_{x}= \pm \pi\right.$ or $\left.k_{y}= \pm \pi\right)$ but deviate slightly from them as schematically shown in Fig. 6(a). This 'shift' is quantified by $\theta_{\mathrm{Y}}($ or $\Delta \eta$ ) as defined in the figure. It has been reported that $\theta_{\mathrm{Y}} \sim 3.2^{\circ}, 2.5^{\circ}$ (or $\Delta \eta \sim 0.0067,0.0052$ ) for $\delta=0.12,0.13,{ }^{14}$ ) respectively. (Hereafter, we use $\Delta \eta$ as our notation.) In the present context, the 'shift' can be understood in terms of the anisotropy of the second n.n. transfer integrals in the orthorhombic crystal structure as follows. 
In the low-temperature orthorhombic (LTO1) structure, $\mathrm{CuO}_{6}$ octahedra tilt around the [110] axis (tetragonal notation) about $\theta=3 \sim 4^{\circ 20)}$ at $\delta=0.10 \sim 0.15$. As a result, (i) the distance between the second n.n. $\mathrm{Cu}$ sites becomes longer along [1]̄0] while it gets shorter along [110], ${ }^{20)}$ and (ii) the alternate buckling of O atoms occurs along [1]0] while the uniform buckling occurs along [110]. Hence, the wave function of the Zhang-Rice singlet ${ }^{21)}$ becomes spatially anisotropic, which leads to an anisotropy between $F_{\|}{ }^{\prime}$ (parallel to [110]) and $F_{\perp}{ }^{\prime}$ (perpendicular to [110]), namely $\left|F_{\|}{ }^{\prime}\right|>\left|F_{\perp}{ }^{\prime}\right|$. The effect (i) will produce the anisotropy of about $1 \sim 2 \%$, if we assume $F^{\prime} \propto t_{p p}$ with $^{22)} t_{p p} \propto r^{-2}$ where $t_{p p}$ is the hopping integral between the n.n. $\mathrm{O}$ sites in a $\mathrm{CuO}_{2}$ plane with distance $r$ (the distance between the second n.n. $\mathrm{Cu}$ sites is $2 r$ ). As for the effect (ii), the degree of the anisotropy is expected to be at least more than $1 \sim 2 \%$, if we assume $F_{\perp}{ }^{\prime} / F_{\|}{ }^{\prime}>\left(1-3.78 \tan ^{2} \theta\right)$ where the form on the right-hand side was used to estimate the effect of the (uniform) buckling of $\mathrm{O}$ atoms on the hopping integral. ${ }^{23)}$ We thus put

$$
F_{\perp}^{\prime}=\gamma F_{\|}^{\prime}(\gamma \leq 1)
$$

We first calculate $\chi_{0}(\boldsymbol{q})$ at $\delta=0.15$ for various $\gamma$. When $\gamma=1, \chi_{0}(\boldsymbol{q})$ takes maxima at $\left(\pi, \pi \pm 2 \pi \eta^{y}\right)$ and $\left(\pi \pm 2 \pi \eta^{x}, \pi\right)$ for the $\mathrm{q}-1 \mathrm{dFS}(\mathrm{x}) .\left(\eta^{x}=0.092\right.$ and $\left.\eta^{y}=0.113.\right)$ With decreasing $\gamma$, the positions shift to $\left(\pi \mp 2 \pi \Delta \eta^{x}, \pi \pm 2 \pi \eta^{y}\right)$ and $\left(\pi \pm 2 \pi \eta^{x}, \pi \mp 2 \pi \Delta \eta^{y}\right)$ where both $\Delta \eta^{x}$ and $\Delta \eta^{y}$ are positive. ${ }^{24)}$ Such a shift is consistent with Fig. 6(a). The 'shift' for the $\mathrm{q}-1 \mathrm{dFS}(\mathrm{y})$ is obtained by reflecting that for the $\mathrm{q}-1 \mathrm{dFS}(\mathrm{x})$ about the $q_{y}=q_{x}$ axis, and is also consistent with Fig. 6(a). This is because, while the shape of $\mathrm{q}-1 \mathrm{dFS}(\mathrm{y})$ is rotated by $90^{\circ}$, the tilting axis is not. In Fig. 6(b), we show $\Delta \eta^{x}$ and $\Delta \eta^{y}$ as a function of $\gamma$ where both $\Delta \eta^{x}$ and $\Delta \eta^{y}$ increase with decreasing $\gamma$. At $\delta=0.10$, we find that the value of $\Delta \eta^{x}$ is almost the same as that for $\delta=0.15$ at each $\gamma$, while the value of $\Delta \eta^{y}$ becomes about 1.5 times larger. Thus, at $\delta \approx 0.12 \sim 0.13$, the values of $\Delta \eta^{x}$ and $\Delta \eta^{y}$ will be comparable to the experimental values when the anisotropy of $F^{\prime}$ is $\sim 10 \%(\gamma \sim 0.9)$. This value of anisotropy is reasonable because, as estimated in the previous paragraph, the anisotropy due to the effects (i) and (ii) is expected to be at least more than $2 \sim 4 \%$ in total. Therefore, fermiology can also explain the 'shift' semiquantitatively in terms of the anisotropy of the second n.n. transfer integrals $F$ ' caused by the LTO1 structure. In the LTT structure, the 'shift' is not expected in the present context because there is no anisotropy in $F^{\prime}$. This prediction can be tested experimentally on Nd-doped LSCO.

We have proposed a q-1dFS scenario for LSCO. Whether the same scenario applies to other high- $T_{c}$ cuprates, such as YBCO and Bi2212, is one of the questions we are trying to 
answer. ${ }^{13)}$ In addition, any relation of the present $\mathrm{q}-1 \mathrm{dFS}$ to the 'charge stripe' picture $^{9-11)}$ has not been obtained in our current analysis and is left as a future problem. As a test for the present picture, observations of (i) both 'outer FS' and 'inner FS', or (ii) both $\eta^{x}$ and $\eta^{y}$ will be crucial. As for (ii), however, we note some possibilities such as (a) $\eta^{x}$ and $\eta^{y}$ are too close compared to the peak width, and (b) IC-AF with either $\eta^{x}$ or $\eta^{y}$ develops due to the instability toward static IC-AF ordering, or due to the coupling ${ }^{25,26)}$ to a possible vertical (or horizontal) charge stripe.

In summary, we have proposed, for LSCO, a scenario that a q-1dFS is realized in each $\mathrm{CuO}_{2}$ plane whose q-1d direction alternates along the $c$-axis. We have shown that the observed FS can be understood as two orthogonal q-1dFSs. On the basis of fermiology, we have also shown that (i) IC-AF fluctuation has two incommensurabilities, $\eta^{x}$ and $\eta^{y}$, and (ii) the 'shift' of sharp IC peaks from the symmetry axis can be understood as coming from the anisotropy of the second n.n. hopping integral in the LTO1 structure. As a test of the present picture, observations of both 'outer FS' and 'inner FS' will be conclusive.

We thank Dr. A. Ino, Dr. K. Kuroki and especially Professor H. Fukuyama for fruitful discussions. H. Y. also thanks Dr. H. Kimura and Mr. T. Yoshida for stimulating discussions. This work is supported by a Grant-in-Aid for Scientific Research from Monbusho. 


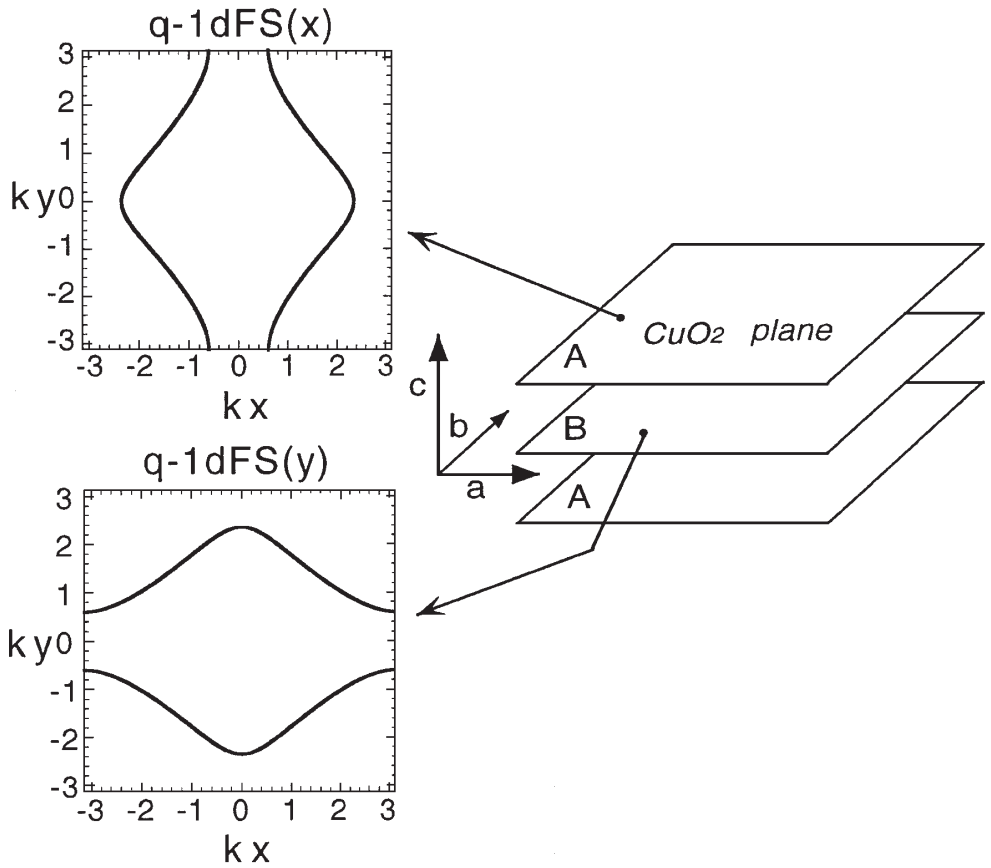

Fig. 1. Proposed quasi-one-dimensional (q-1d) FSs, q-1dFS(x) and q-1dFS(y), and their alternate stacking along the c-axis.
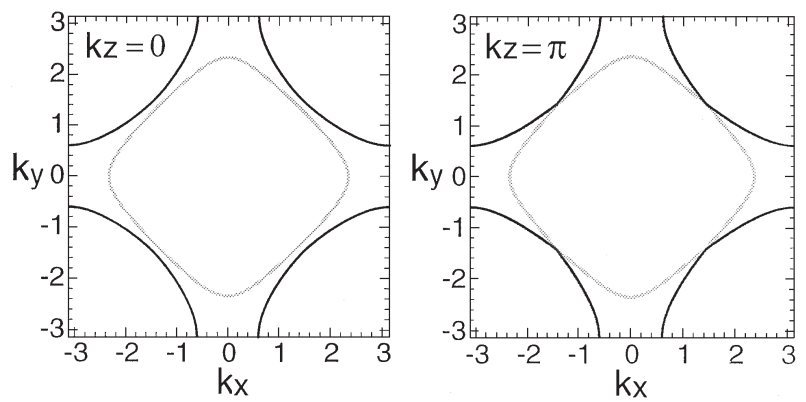

Fig. 2. The theoretical FS at $k_{z}=0$ and $k_{z}=\pi$. The FS consists of the 'outer FS', shown by the solid line, and the 'inner FS', shown by the gray line. 


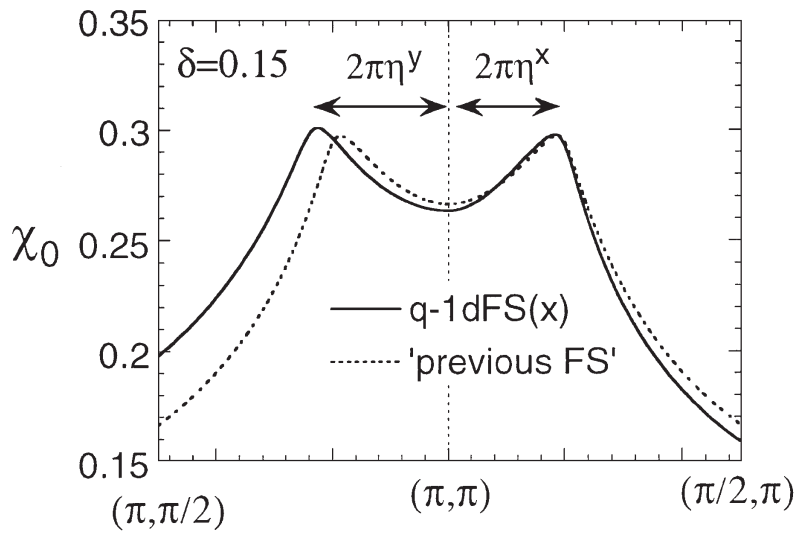

Fig. 3. The $\chi_{0}(\boldsymbol{q})$ for the $\mathrm{q}-1 \mathrm{dFS}(\mathrm{x})$ at $\delta=0.15$. The peak positions of $\chi_{0}(\boldsymbol{q})$ are measured from $(\pi, \pi)$ as $\eta^{x}$ or $\eta^{y}$. For comparison, the result for the 'previous FS' (see the text) is shown by a dashed line.

\section{(a)}

(b)
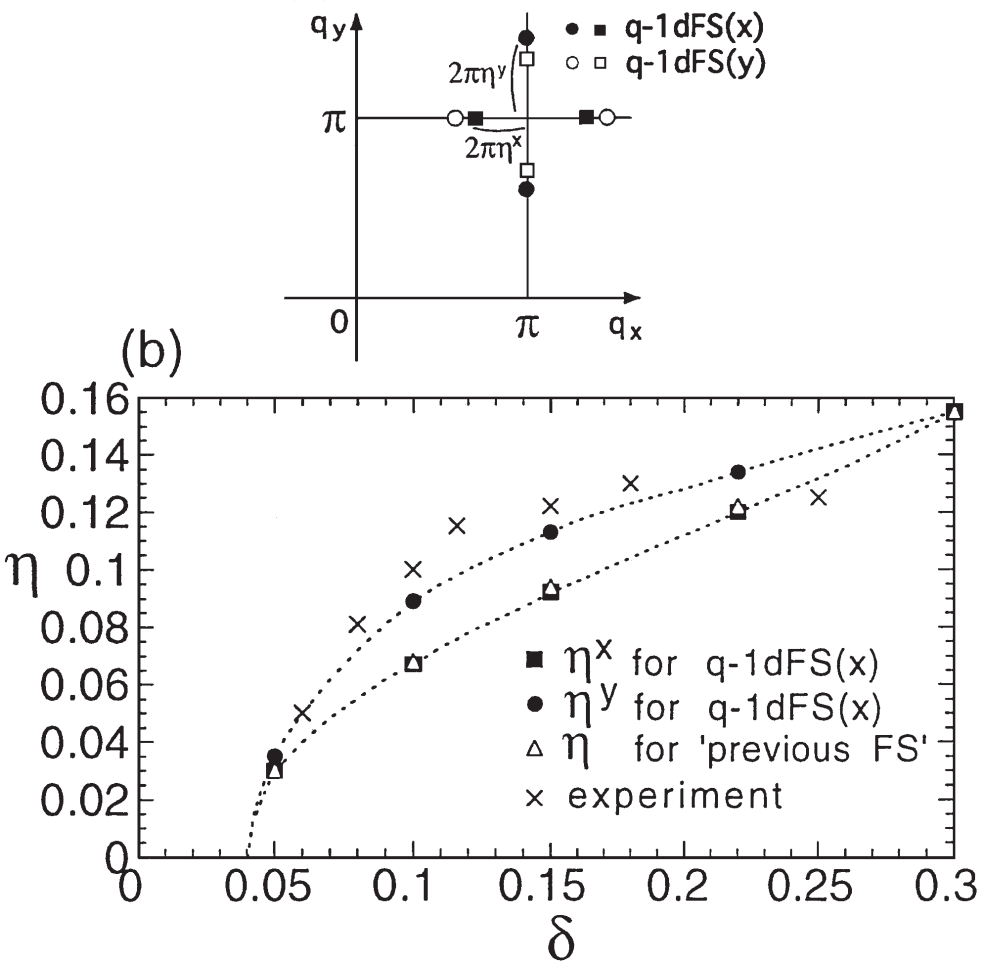

Fig. 4. (a) Schematic figure of IC peak positions for the q-1dFS(x) (filled symbols) and the q-1dFS(y) (open symbols). Note that the positions for the $\mathrm{q}-1 \mathrm{dFS}(\mathrm{y})$ are obtained by the $90^{\circ}$ rotation from those for $\mathrm{q}-1 \mathrm{dFS}(\mathrm{x})$. (b) The $\eta^{x}$ and $\eta^{y}$ for $\mathrm{q}-1 \mathrm{dFS}(\mathrm{x})$ as a function of $\delta$. Dashed lines are guides for the eye. For comparison, the result for the 'previous FS' and experimental values are also shown. 


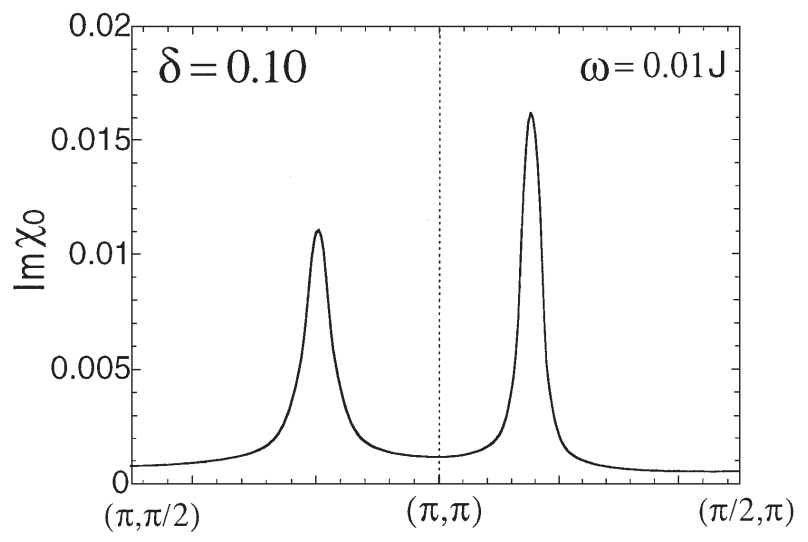

Fig. 5. The sharp IC peaks of $\operatorname{Im} \chi_{0}(\boldsymbol{q}, \omega)$ at $\delta=0.10$ and $\omega=0.01 J$.

(a)

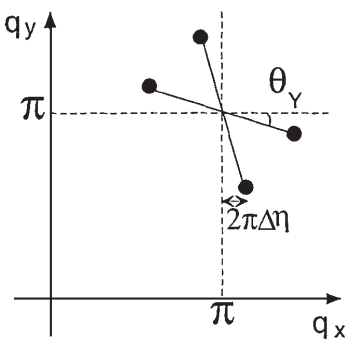

(b)

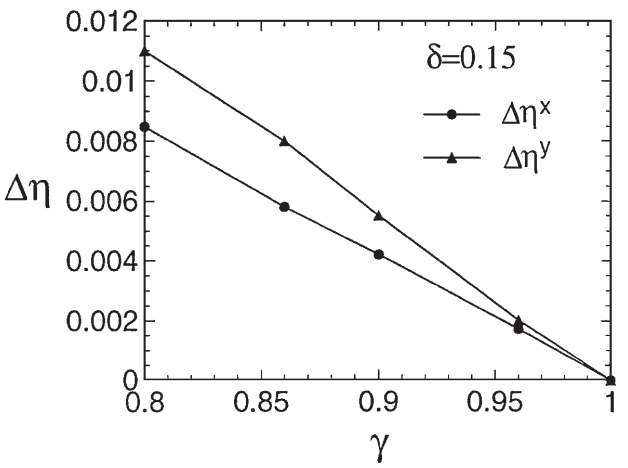

Fig. 6. (a) Location of the elastic peaks observed by neutron scattering. They slightly shift away from the symmetry axis ( $k_{x}= \pm \pi$ or $k_{y}= \pm \pi$ ), and this 'shift' is quantified by $\Delta \eta$ or $\theta_{\mathrm{Y}}$. (b) The $\Delta \eta^{x}$ and $\Delta \eta^{y}$ at $\delta=0.15$ as a function of $\gamma$. For the definition of $\Delta \eta^{x}$ and $\Delta \eta^{y}$, see the text. 


\section{References}

1) K. Yamada, C. H. Lee, K. Kurahashi, J. Wada, S. Wakimoto, S. Ueki, H. Kimura, Y. Endoh, S. Hosoya, G. Shirane, R. J. Birgeneau, M. Greven, M. A. Kastner and Y. J. Kim: Phys. Rev. B 57 (1998) 6165 and references therein.

2) J. Rossat-Mignod, J. P. Regnault, C. Vettier, P. Burlet, J. Y. Henry and G. Lapertot: Physica B 169 (1991) 58.

3) J. Rossat-Mignod, J. P. Regnault, C. Vettier, P. Bourges, P. Burlet, J. Bossy, J. Y. Henry and G. Lapertot: Physica C 185 - 189 (1991) 86.

4) Qimiao Si, Yuyao Zha, K. Levin and J. P. Lu: Phys. Rev. B 47 (1993) 9055.

5) T. Tanamoto, H. Kohno and H. Fukuyama: J. Phys. Soc. Jpn. 62 (1993) 717.

6) A. Ino, C. Kim, T. Mizokawa, Z.-X. Shen. A. Fujimori, M. Takaba, K. Tamasaku, H. Eisaki and S. Uchida: J. Phys. Soc. Jpn. 68 (1999) 1496.

7) We consider $\delta$ is equal to $\mathrm{Sr}^{+2}$-content, $x$.

8) K. Kuroki, R. Arita and H. Aoki: Phys. Rev. B 60 (1999) 9850.

9) J. M. Tranquada, B. J. Sternlieb, J. D. Axe, Y. Nakamura and S. Uchida: Nature 375 (1995) 561.

10) J. M. Tranquada, J. D. Axe, N. Ichikawa, Y. Nakamura, S. Uchida and B. Nachumi: Phys. Rev. B 54 (1996) 7489.

11) J. M. Tranquada, J. D. Axe, N. Ichikawa, A. R. Moodenbaugh, Y. Nakamura and S. Uchida: Phys. Rev. Lett. 78 (1997) 338.

12) H. Yamase, H. Kohno and H. Fukuyama: submitted to Physica C.

13) H. Yamase and H. Kohno: submitted to J. Phys. Soc. Jpn.

14) H. Kimura: private communications.

15) Y. S. Lee, R. J. Birgeneau, M. A. Kastner, Y. Endoh, S. Wakimoto, K. Yamada, R. W. Erwin, S.-H. Lee and G. Shirane: Phys. Rev. B 60 (1999) 3643.

16) T. R. Thurston, R. J. Birgeneau, D. R. Gabbe, H. P. Jenssen, M. A. Kastner, P. J. Picone, N. W. Preyer, J. D. Axe, P. Böni, G. Shirane, M. Sato, K. Fukuda and S. Shamoto: Phys. Rev. B 39 (1989) 4327.

17) W. E. Pikett: Rev. Mod. Phys. 61 (1989) 433.

18) X. J. Zhou, P. Bogdanov, S. A. Kellar, T. Noda, H. Eisaki, S. Uchida, Z. Hussain and Z.-X. Shen: Science 286 (1999) 268.

19) S. Wakimoto, G. Shirane, Y. Endoh, K. Hirota, S. Ueki, K. Yamada, R. J. Birgeneau, M. A. Kastner, Y. S. Lee, P. M. Gehring and S. H. Lee: Phys. Rev. B 60 (1999) R769.

20) P. G. Radaelli, D. G. Hinks, A. W. Mitchell, B. A. Hunter, J. L. Wagner, B. Dabrowski, K. G. Vandervoort, H. K. Viswanathan and J. D. Jorgensen: Phys. Rev. B 49 (1994) 4163.

21) F. C. Zhang and T. M. Rice: Phys. Rev. B 37 (1988) 3759.

22) W. A. Harisson: Electronic Structure and the Properties of Solids (Freeman, New York, 1980).

23) B. Normand, H. Kohno and H. Fukuyama: Phys. Rev. B 53 (1996) 856.

24) Strictly speaking, the values of $\eta^{x}$ and $\eta^{y}$ become larger by about $1 \sim 2 \%$ with decreasing $\gamma$.

25) K. Miyake and O. Narikiyo: J. Phys. Soc. Jpn. 63 (1994) 2042.

26) H. Yamase, H. Kohno, H. Fukuyama and M. Ogata: J. Phys. Soc. Jpn. 68 (1999) 1082. 